shown a high incidence of chronic arachnoiditis (diagnosed by radiological criteria). For some time it has been a recognised risk of the use of the oil-soluble media (such as iophendylate). Unfortunately aspiration of the contrast medium after the examination does not necessarily give protection. The hope that water-soluble substances would prove innocuous has proved false, and many cases have been described after the use of iothalamate meglumine (Conray) and iocarmate meglumine (Dimer-X). So far, however, sodium calcium medetate (metrizamide) has proved to be safe $^{6}$ : it has been more extensively tested than any other contrast medium but no case of arachnoiditis has yet been attributed to its use.

The subdural space is very sensitive to irritants, even as benign as blood, and insufficient attention has been paid to subdural leakage as a cause of arachnoiditis. Could constrictive arachnoiditis really be a reaction in the subdural space? If this is so-and there is an association ${ }^{3}$ between "difficult" lumbar punctures and arachnoiditis-then doctors should give more consideration to postponing a radiological contrast examination in any case in which puncture of the subarachnoid space has not been clean and immediate. For the time being metrizamide seems to be the spinal contrast medium of choice, while air should be used whenever possible as an alternative, especially in children.

\footnotetext{
${ }^{1}$ Elkington, J St C, Brain, 1936, 59, 181.

${ }^{2}$ Elkington, J St C, in Modern Trends in Neurology, ed A Feiling, p 149. London, Butterworth, 1951.

3 Shaw, M D M, Russell, J A, and Grossart, K W, fournal of Neurology, Neurosurgery, and Psychiatry, 1978, 41, 97.

- Bernat, J L, Fournal of Neurology, Neurosurgery, and Psychiatry, 1976, 39, 1124.

5 Ransford, A C, and Harries, B J, fournal of Bone and foint Surgery, 1972, 54, 656 .

${ }^{6}$ Hansen, E B, Fahrenkrieg, A, and Praestholm, J, British fournal of Radio$\log y, 1978, \mathbf{5 1}, 321$
}

\section{Growing fractures of the skull}

In 1816 John Howship ${ }^{1}$ described a 9-month-old child who had developed partial absorption of the right parietal bone after a blow to the head. The names used since then to describe the condition include meningocele spuria, ${ }^{2}$ traumatic cephalhydrocele, ${ }^{3}$ traumatic ventricular cyst, ${ }^{4}$ and leptomeningeal cyst. ${ }^{5}$ More recently the term "growing fracture" has become accepted. ${ }^{6}$ There are four characteristic features: a skull fracture in infancy or early childhood; a dural tear; brain injury beneath the fracture; and subsequent enlargement of the fracture to form a cranial defect. ${ }^{7}$

Growing fracture is uncommon; one American series documented only seven patients operated on in 20 years, ${ }^{8}$ but a recent paper from London has reported 10 patients seen in 10 years. ${ }^{9}$ Most had had their initial injury before the age of 1 year and almost all by 3 ; the fracture was commonly in the parietal region. The time between injury and diagnosis was generally short; the mean interval was 15 months, the shortest two and the longest 63 months. The diagnoses in this series were made earlier than in those previously reported, ${ }^{8}$ probably because surgeons are more aware of the condition now that the criteria for its diagnosis have been established.

Patients most often present with a pulsatile swelling of the scalp, though seizures and neurological deficits such as hemiplegia may occur. Neuroradiological investigations show dilatation of the ipsilateral ventricle with midline displacement to the same side. A porencephalic cyst is generally present and often communicates with the lateral ventricle. ${ }^{8}$ In the past these features have been shown by pneumoencephalography, but CAT scanning is likely to prove valuable in making the diagnosis: it will show up not only the underlying ventricular dilatation and cystic cavity but also the bony defect.

While a growing fracture always implies severe trauma at a time of maximum growth of both the skull and the underlying brain we do not know why the fracture should continue to enlarge. One possibility is that leptomeningeal cysts (fluidfilled spaces between the pia mater and the arachnoid membrane) cause the bone erosion and enlargement. These cysts are, however, rare, and at operation the pia and arachnoid membranes are generally found to be matted together and stuck over the underlying atrophic brain. ${ }^{9}$ More probably the fracture continues to grow both because ventricular size is increasing and because the dura is torn. Nevertheless, the relative contribution of these two factors is not clear and the evidence from experimental work is conflicting. ${ }^{1011}$ After a head injury, changes occur in the dynamics of formation and flow of cerebrospinal fluid, and these may increase its pressure. If the ventricle is enlarged any increase in this pressure will produce a disproportionate rise in the subdural stress; and with a dural defect this increase in stress will cause a pulsatile swelling, which will then erode the bone and increase the defect.

The growth of the fracture, then, results partly from the dural defect. Hence the key to its control is an operation to ensure a watertight closure of the dura. The bony defect itself can then be closed with a split rib graft or with acrylic (which has been used more often in recent years). The outlook after surgery is good: of eight children followed for up to 10 years, five were normal, two had a mild hemiparesis, and only one was slightly mentally backward. The earlier the diagnosis is made the better, but since such fractures are rare repeated radiographs are not justified as a routine in the follow-up of every skull fracture in childhood. Suspicion is indicated only in infants with a parietal fracture with notable separation of the bone edges.

\footnotetext{
${ }^{1}$ Howship, J, Practical Observations in Surgery and Morbid Anatomy, p 494. London, Longman, Hurst, Reece, Ormond-Brown, 1816.

2 Bilroth, T, Archiv für Klinische Chirurgie, 1862, 3, 398.

3 Conner, P S, American fournal of the Medical Sciences, 1884, 88, 103.

4 Trotter, W A, A System of Surgery, ed C C Choyce and J M Beattie, vol 3, p 536. New York, Hoeber, 1923.

5 Dyke, C G, Diagnostic Roentgenology, ed R Golden, p 116. Baltimore, Williams and Hopkins, 1948.

${ }^{6} \mathrm{Pia}, \mathrm{H} \mathrm{W}$, and Tonnis, W, Zentralblatt für Neurochirurgie, 1953, 13, 1.

7 Lende, R A, and Erickson, T C, fournal of Neurosurgery, 1961, 18, 479.

8 Taveras, J M, and Ransohoff, J, Fournal of Neurosurgery, 1953, 10, 233.

${ }^{-}$Kingsley, D, Till, K, and Hoare, R, Fournal of Neurology, Neurosurgery and Psychiatry, 1978, 41, 312.

10 Goldstein, F, et al, fournal of Neurosurgery, 1967, 27, 541.

11 Lende, R A, Neuroradiology, 1974, 7, 119.
}

\section{Quest for excellence}

It looks as though we have got postgraduate medical education in Britain about right. There are, of course, things that need watching. Some training programmes are too inflexible, and general practitioners have set their own precedent by having theirs embodied in an Act of Parliament. The method of assessing posts for training has proved expensive and time 